\title{
Flutuação temporal de cianotoxinas (Microcistina- LR) no rio Tapajós (Santarém, Amazônia-Brasil)
}

Temporal fluctuation of cyanotoxins (Microcystin-LR) in the Tapajós river (Santarém, AmazonBrazil)

\author{
S. C. F. Silva ${ }^{1 *}$; J. R. P. Peleja ${ }^{2} ;$ S. Melo ${ }^{1}$ \\ ${ }^{I}$ Programa de Pós-Graduação Sociedade, Natureza e Desenvolvimento (PPGSND)/ Universidade Federal do Oeste \\ do Pará (UFOPA), 68040-070, Santarém-PA, Brasil \\ ${ }^{2}$ Instituto de Ciências e Tecnologia das Águas (ICTA)/Laboratório de Biologia Ambiental, Universidade Federal do \\ Oeste do Pará (UFOPA), 68040-070, Santarém-PA, Brasil \\ *sameacibele@yahoo.com.br \\ (Recebido em 19 de abril de 2019; aceito em 19 de agosto de 2019)
}

\begin{abstract}
O relato de produção de microcistinas tem aumentado nas últimas décadas e é um problema preocupante em diversos ambientes aquáticos naturais e antropizados, em especial nos lênticos. Poucos são os relatos de presença de cianotoxinas em ambientes lóticos, entre estes está o registro no rio Tapajós. O presente estudo teve por objetivo analisar a ocorrência de microcistina em amostras de água coletadas em escala quinzenal no rio Tapajós (Santarém, PA, Brasil) em uma região de recreação ao longo de um ciclo anual. A concentração de microcistina foi quantificada pelo método Imunológico-ELISA. Ao longo do estudo foram registradas a ocorrência de 11 táxons de cianobactérias, sendo Microcystis, Dolichospermum e Planktothrix os gêneros que apresentam espécies potencialmente produtoras de cianotoxinas. A presença de microcistina foi constatada em todo o período amostrado e sua concentração máxima esteve associada à presença de espécies do gênero Microcystis sp. Embora os valores constatados sejam inferiores aos considerados como limite para o uso recreativo, a ocorrência de gêneros de cianobactérias, potencialmente produtores de cianotoxinas, e o fato da detecção de microcistina em todo o período de estudo, torna-se importante os estudos sobre este grupo de microrganismos no rio Tapajós. Temporalmente, não foi constatado diferenças significativas dos níveis de microcistina em relação ao regime pluviométrico e ao pulso de inundação.

Palavras-chave: Cianobacterias, Amazônia, ambiente lótico.
\end{abstract}

The report of microcystins production has increased in the last decades and is a worrying problem in several natural and anthropized aquatic environments, especially in the lentic ones. There are few reports of the presence of cyanotoxins in lotic environments, among which is the record in the Tapajós river. The present study had the objective of analyzing the occurrence of microcystin in water samples collected on a biweekly scale in the Tapajós river (Santarém, PA, Brazil) in a recreation region along an annual cycle. The microcystin concentration was quantified by the Immunological-ELISA method. During the study the occurrence of 11 taxa of cyanobacteria was recorded, being Microcystis, Dolichospermum and Planktothrix the genera that present species potentially producing cyanotoxins. The presence of microcystin was observed throughout the sampled period and its maximum concentration was associated with the presence of species of the genus Microcystis sp. Although the values found are lower than those considered as a limit imposed for recreational use, the occurrence of genera of cyanobacteria, potentially cyanotoxin producers, and the fact of microcystin detection throughout the study period, it is important to study this group of microorganisms in the Tapajós river. Temporally, there were no significant differences in microcystin levels in relation to the pluviometric regime and to the flood pulse.

Keywords: Cyanobacteria, Amazon region, lotic environment.

\section{INTRODUÇÃO}

Cianotoxinas são compostos secundários sintetizados por cianobactérias os quais possuem efeitos biológicos tóxicos. A toxicidade de florações de cianobactérias pode apresentar variação temporal e também espacial, provavelmente decorrentes de alterações na proporção de cepas tóxicas e não tóxicas na população [1,2]. As cianotoxinas podem afetar a saúde humana, tanto através da ingestão de água contaminada, como por contato direto em atividades de recreação. Estas substâncias químicas podem ser incorporadas nos diferentes níveis da cadeia trófica e 
ocasionar intoxicação, até mesmo provocar a morte de humanos [3, 4]. Assim, as toxinas de cianobactérias podem causar sérios problemas ambientais, bem como comprometer o uso da água doce para fins de consumo humano direto, irrigação, pesca e fins recreativos [5].

De acordo com sua ação farmacológica, as principais classes de cianotoxinas são: dermatotoxinas, neurotoxinas e hepatotoxinas. As dermatotoxinas são lipopolissacarídeos (LPS) integrantes da parede celular de todas as cianobactérias e bactérias Gram negativas [6]. O contato com o LPS durante o contato recreacional, pode causar irritação da pele humana, bolhas e distúrbios gastrointestinais [7].

As neurotoxinas atuam no sistema nervoso mesmo em baixas concentrações. São alcalóides produzidos principalmente pelos gêneros: Dolichospermum, Aphanizomenon, Oscillatoria, Trichodesmium, Lyngbya e Cilindrospermopsis [8]. São de rápida atuação, e os sintomas de intoxicação aguda em mamíferos são paralisia, hipotensão, dispnéia e falência respiratória [9].

Já as hepatotoxinas são heptapeptídeos que agem principalmente no fígado e apresentam uma ação mais lenta comparadas as neurotoxinas, incluem peptídeos cíclicos que compreendem as microcistinas e as nodularinas, e um alcalóide, a cilindrospermopsina que também age em outros órgãos [6, 10]. As espécies produtoras de hepatotoxinas pertencem aos gêneros Microcystis, Dolichospermum, Nodularia, Oscillatoria, Nostoc, Cylindrospermopsis, Umezakia, Aphanizomenon, Hapalosiphon, Anabaenopsis, Trichodesmium, Synechococcus, Snowella [11, 12].

As microcistinas são heptapeptídeos que possuem em sua composição cinco aminoácidos constantes e dois variantes $[13,14]$. Essas toxinas são caracterizadas pela presença de 5 Daminoácidos e 2 L-aminoácidos $[14,15]$. Para a nomenclatura das microcistinas acrescentam-se as iniciais dos dois $\mathrm{L}$ aminoácidos após o nome da toxina. Atualmente mais de 100 diferentes tipos de microcistinas foram descritas [14, 16, 17], porém a variante mais comum encontrada em amostras ambientais é a microcistina-LR (MC-LR), que contém uma L-leucina e uma L-arginina nas posições dos aminoácidos $[18,19]$. Estudos mostram que as microcistinas também podem afetar outros órgãos além do fígado, incluindo o cólon, os rins e os pulmões [10, 20, 21].

A comprovação da toxicidade de uma população de cianobactérias em um determinado corpo d'água não implica necessariamente perigo ambiental e humano enquanto as células permanecerem finamente dispersas, todavia o desenvolvimento massivo, com formação de escuma na superfície representam riscos para a saúde [22]. As cianotoxinas em águas de uso recreativo podem constituir um perigo grave para a saúde humana devido à exposição por ingestão acidental de água contaminada, inalação e por contato dérmico [23, 24, 25, 26]. O valor tolerado para exposição recreativa é de no máximo $10 \mu \mathrm{g} . \mathrm{L}^{-1}$ para MC-LR [22, 27]. A OMS estipulou o valor provisório máximo de $1,0 \mu \mathrm{g} . \mathrm{L}^{-1}$ para MC-LR como limite de segurança para água potável e o valor de tolerância diária ingerida (TDI) de $0,04 \mu \mathrm{g}$ de MC-LR por quilograma $(\mathrm{Kg})$ de peso corpóreo por dia [28].

A legislação brasileira de controle e vigilância da qualidade da água para consumo humano e seu padrão de potabilidade determina o valor máximo permitido (VMP) de $1,0 \mu \mathrm{g} . \mathrm{L}^{-1}$ para

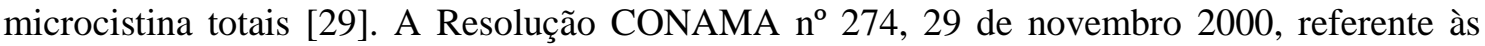
condições de balneabilidade, estabelece restrições à recreação de contato primário quando verificada a ocorrência de florações de algas e considera passível de interdição pelos órgãos de controle ambiental trechos dos corpos d'água em que ocorram toxicidade ou formação de escuma decorrente de florações de algas [30]. Adicionalmente, a Resolução CONAMA n ${ }^{\circ}$ 357, de 17 de março de 2005, estabelece VMP para a densidade de cianobactérias de até 20.000, 50.000 e $100.000 \mathrm{cel} / \mathrm{mL}$ para águas doce classe 1,2 e 3 respectivamente, destinadas a recreação de contato primário [31].

Em diversos países, as cianobactérias tóxicas têm sido descritas como produtoras de microcistinas, com destaque para os gêneros Microcystis, Dolichospermum, Nostoc e Planktothrix [32, 33, 34], por serem as mais frequentes em corpos d'água em todo o mundo [30]. Eutrofização e mudanças climáticas são dois processos que promovem a proliferação e expansão de florações nocivas de cianobactérias [5, 12]. Na América do Sul os corpos d'água são particularmente vulneráveis às florações e presença de microcistina em função de fatores ambientais favoráveis [34, 35, 36]. 
O crescimento de atividades intrinsecamente humanas tais como a mineração, a extração de madeira e a abertura de pastagem, que compõem, juntamente com a expansão do cultivo de grãos, um mosaico de usos diferenciados do espaço amazônico vêm alterando, de forma radical, a dinâmica tradicional de ocupação da Amazônia brasileira [37, 38]. Em Santarém a expansão da fronteira agrícola, assim como a distribuição espacial das áreas desmatadas foi registrado nos mapeamentos referentes ao período de 2000 a 2013 [37, 39]. Tais modificações são preocupantes visto que os corpos d'água são afetados pelas ações do uso da terra e a ocupação humana. $\mathrm{O}$ uso e ocupação do solo de maneira desordenada contribui para o enriquecimento nutricional dos corpos d'água e favorece a floração de cianobactérias potencialmente tóxicas [16].

Outro fator que pode estar associado a ocorrência de florações de cianobactérias é a disponibilidade de carbonatos e bicarbonatos disponível na água que podem ter suas concentrações aumentadas associada ao aumento da concentração de $\mathrm{CO} 2$ atmosférico [40]. Estes autores verificaram que em condições de laboratório e de campo que o aumento na concentração de carbono induz uma rápida mudança na composição fenotípica em florações de cianobactétrias tóxicas. A eficiência no uso de bicabornato foi sugerido como uma ação que pode determinar a dominância e o sucesso competitivo de cianobactérias potencialmente tóxica, tais como Microcystis [41].

Os gêneros Aphanizomenon, Aphanocapsa, Dolichospermum, Hapalosiphon, Jaaginema, Microcystis, Nostoc, Oscillatoria, Planktothrix, Raphidiopsis, Radiocystis. e Snowella, potencialmente produtores de cianotoxinas [42], já foram registrados no Estado do Pará [43, 44]. Neste estado, foram isoladas cepas, de Microcystis, e Radiocystis, produtoras de microcistinas em água bruta encontrada no reservatório de abastecimento público, Utinga, PA [43, 44]. Em especial para o rio Tapajós, no município de Santarém, foram registrados Anabaena e Microcystis, com produção de microcistina [45]. Destaca-se que o referido trabalho se baseou em um único período de coletas em cinco pontos amostrais no mês de março de 2007.

O rio Tapajós, é utilizado como uma grande hidrovia, utilizado para pesca e área de lazer, sendo suas praias um atrativo turístico. No entanto, não é raro visualizar alteração da cor da sua água devido a ocorrência de florações de cianobactérias, florações estas que tiveram a presença de microcistina confirmada [45]. Considerando a importância do rio Tapajós e, que a presença de cianotoxinas/microcistinas pode conferir danos à comunidade aquática e à população humana que faz uso direto ou indireto de suas águas, torna-se relevante estudos de visam avaliar possível ocorrência de florações tóxicas. Diante do exposto, o presente estudo teve por objetivo analisar a presença de MC-LR em amostras de água obtidas em escala quinzenal no rio Tapajós em uma região utilizada para recreação na área urbana de Santarém/Pará.

\section{MATERIAL E MÉTODOS}

O rio Tapajós é formado pela junção dos rios Juruena e Teles Pires e é afluente da margem direita do rio Amazonas na frente da cidade de Santarém. É um rio de águas claras, com transparência superior a 2,5 m, e coloração aparente esverdeada, devido à presença significativa de carbonatos, além de pouco material particulado em suspensão [46, 47, 48]. A parte inferior da bacia do rio Tapajós cobre cerca de $130.370 \mathrm{~km}^{2}$ e em seu trecho mais baixo, na região de Aveiro até a sua foz em Santarém. O rio Tapajós apresenta-se como uma planície aluvial bastante larga, formando um sistema fluvial com característica semi-lêntica [49, 38].

É comum a ocorrência de florações de cianobactérias (localmente conhecidas como limo) que podem ser visíveis em vários momentos ao longo do ano em sua porção próxima a Santarém. $\mathrm{O}$ primeiro registro de elevadas densidades de cianobactérias no rio Tapajós data da década de 60 do século passado, e a elevada produtividade primária do fitoplâncton foi atribuída a alta disponibilidade de luz em virtude da elevada transparência da água deste rio [50, 51].

As amostragens foram efetuadas no rio Tapajós, em um único ponto amostral $\left(02^{\circ} 25^{\prime} 08.7^{\prime \prime}\right.$, 054 45’39.0”O), entre as praias do Maracanã e Juá, à montante da orla urbana do município de Santarém, no período de outubro de 2010 a setembro de 2011 em escala quinzenal, exceto nos meses de janeiro e fevereiro de 2011.

As amostras de água bruta foram obtidas a cerca de $30 \mathrm{~cm}$ de profundidade do rio, a partir da proa de um bote de madeira. Para as quantificações de MC-LR, clorofila- $a$, os nutrientes 
dissolvidos: amônia, nitrato, nitrito, e orto-fosfato, as amostras foram armazenadas em garrafas escuras de polietilenotereftalato (PET) previamente identificadas, e mantidas à $4^{\circ} \mathrm{C}$. As análises ocorreram no mesmo dia, exceto quantificação da MC-LR, em que as amostras foram congeladas. Para a identificação das espécies de cianobactérias presentes no ambiente foram obtidas amostras com rede de plâncton com abertura de malha de $20 \mu \mathrm{m}$ e fixadas em formol a $4 \%$. A riqueza em espécies foi estimada pelo número de táxons por amostra. No momento da coleta foi estimado a transparência pela profundidade de extinção do disco de Secchi.

Os valores de condutividade elétrica e sólidos totais dissolvidos foram obtidos através do condutivímetro WWR modelo 2052, conforme preconizado pela AWWA [52], oxigênio dissolvido e temperatura com um oxímetro YSI modelo 58 e pH, com um potenciômetro de $\mathrm{pH}$ portátil marca OAKTON, modelo WD -35634-00 [52].

No Laboratório, as amostras para quantificação de amônia, nitrato, nitrito, e orto-fosfato foram filtradas em filtros de fibra de vidro $(0,45 \mu \mathrm{m}$, Gelman Sciences), pelo método colorimétrico com o auxílio de um colorímetro de bancada da marca Smart, conforme AWWA (2005) [52]. Para a determinação da clorofila- $a$, um volume de amostras entre 200 a $2000 \mathrm{~mL}$ foi filtrado em filtros de fibra de vidro $(0,45 \mu \mathrm{m}$, Gelman Sciences) e a análise seguiu o método tricromático de Strickland e Pearsons (1968) [53], com a utilização de um espectrofotômetro de absorção molecular, modelo UV1201-Shimadzu nos comprimentos de onda de 630, 645 e $665 \mathrm{~nm}$.

Para a determinação das concentrações de MC-LR, as amostras de água bruta passaram pelo processo de gelo/degelo por três vezes, sendo posteriormente filtradas em filtros de fibra de vidro $(0,45 \mu \mathrm{m}$, Gelman Sciences). Na sequência foram analisadas pelo método imunológico-ELISA para quantificação de MC-LR em água por meio do Kit em tubo da marca BeacMCon [54] com quantificação em cubetas com volume de $1,5 \mathrm{ml}$ por espectrofotometria de absorção molecular, em espectrofotômetro modelo UV1201-Shimadzu no comprimento de onda de $450 \mathrm{~nm}$. O método ELISA utiliza anticorpos que foram desenvolvidos contra a MC-LR [55] e baseia-se na fixação de anticorpos às toxinas, de maneira que as enzimas sejam usadas para produzir uma reação promovendo uma alteração de cor.

Os dados pluviométricos e de cota na área de drenagem do rio Tapajós, foram obtidos no site da Agência Nacional de Águas (ANA) pelo Sistema Nacional de Informações sobre Recursos Hídricos (HIDROWEB) estação Santarém, código 17900000. A partir destes foi possível delimitar o regime pluviométrico e o pulso de inundação do período de estudo na região.

Para verificar diferenças entre os níveis de MC-LR quanto ao regime pluviométrico e quanto ao pulso de inundação, foi aplicada a Análise de Variância Unifatorial ( $\mathrm{p}<0,05 \%$ ). A Análise de Correlação linear de Pearson $(\mathrm{p}<0,05 \%)$ foi utilizada para averiguar correlação entre os valores de MC-LR e as variáveis independentes de forma isoladamente. A Análise de Regressão Linear Múltipla avaliou a influência conjunta das variáveis independentes sobre MC-LR ( $p<0,05 \%)$. Todas as análises estatísticas foram efetuadas com o auxílio do Software Statistica, versão 7.1 (Stat Soft., 2005).

\section{RESULTADOS E DISCUSSÃO}

Durante o período de estudo foi detectada a riqueza específica de 11 espécies de cianobactérias, sendo Microcystis (M. aeruginosa e M. wesenbergii) e Synechococcus sp. presentes em todas as amostras analisadas (Tabela 1). Destaca-se que os gêneros Microcystis, Dolichospermum e Planktothrix, têm sido citados na literatura como produtores de MC-LR [6, 32]. Destes, Microcystis e Dolichospermum são os táxons com o maior número de espécies comprovadamente tóxicas ocorrentes no Brasil [56] com ocorrência de espécies tóxicas registradas no estado do Pará $[43,45]$.

Tabela 1: Composição dos táxons de cianobactérias encontrados no baixo rio Tapajós no período de outubro de 2010 a setembro de 2011.

\begin{tabular}{lcccccccccccc}
\hline Táxons/Meses & Out & Nov & Dez & Jan & Fev & Mar & Abr & Mai & Jun & Jul & Ago & Set \\
\hline Aphanothece $\mathrm{sp}$. & $\mathrm{X}$ & & & $\mathrm{x}$ & $\mathrm{x}$ & $\mathrm{x}$ & & $\mathrm{x}$ & & $\mathrm{x}$ & & \\
Chroococcus $\mathrm{sp}$. & $\mathrm{x}$ & $\mathrm{x}$ & $\mathrm{x}$ & & $\mathrm{x}$ & $\mathrm{x}$ & $\mathrm{x}$ & $\mathrm{x}$ & $\mathrm{x}$ & & $\mathrm{x}$ & $\mathrm{x}$
\end{tabular}




\begin{tabular}{|c|c|c|c|c|c|c|c|c|c|c|c|c|}
\hline $\begin{array}{l}\text { Dolichospermum } \\
\text { circinalis }\end{array}$ & $\mathrm{X}$ & $\mathrm{X}$ & $\mathrm{X}$ & $\mathrm{X}$ & $\mathrm{X}$ & $\mathrm{X}$ & $\mathrm{X}$ & & & & $\mathrm{X}$ & $\mathrm{X}$ \\
\hline $\begin{array}{l}\text { Dolichospermum } \\
\text { planctonicum }\end{array}$ & $\mathrm{X}$ & & $\mathrm{X}$ & & & & & & & & & \\
\hline Lyngbya sp. & & $\mathrm{X}$ & $\mathrm{X}$ & $\mathrm{X}$ & & & & & & & & \\
\hline Merismopedia sp. & $\mathrm{X}$ & & $\mathrm{x}$ & $\mathrm{x}$ & $\mathrm{X}$ & $\mathrm{X}$ & $\mathrm{X}$ & $\mathrm{X}$ & $\mathrm{X}$ & $\mathrm{x}$ & $\mathrm{X}$ & \\
\hline Microcystis aeruginosa & $\mathrm{x}$ & $\mathrm{x}$ & $\mathrm{x}$ & $\mathrm{x}$ & $\mathrm{x}$ & $\mathrm{x}$ & $\mathrm{x}$ & $\mathrm{x}$ & $\mathrm{x}$ & $\mathrm{x}$ & $\mathrm{x}$ & $\mathrm{X}$ \\
\hline Microcystis wesenbergii & $\mathrm{x}$ & $\mathrm{x}$ & $\mathrm{x}$ & $\mathrm{x}$ & $\mathrm{x}$ & $\mathrm{x}$ & $\mathrm{x}$ & $\mathrm{x}$ & $\mathrm{x}$ & $\mathrm{x}$ & $\mathrm{x}$ & $\mathrm{x}$ \\
\hline Planktothrix sp. & $\mathrm{x}$ & & $\mathrm{x}$ & & $\mathrm{x}$ & & & & & $\mathrm{x}$ & $\mathrm{x}$ & $\mathrm{x}$ \\
\hline Pseudanabaena sp. & $\mathrm{x}$ & $\mathrm{X}$ & $\mathrm{x}$ & $\mathrm{X}$ & & $\mathrm{X}$ & $\mathrm{x}$ & $\mathrm{X}$ & $\mathrm{x}$ & $\mathrm{x}$ & $\mathrm{x}$ & $\mathrm{x}$ \\
\hline Synechococcus sp. & $\mathrm{x}$ & $\mathrm{x}$ & $\mathrm{x}$ & $\mathrm{x}$ & $X$ & $\mathrm{x}$ & $\mathrm{X}$ & $\mathrm{X}$ & $\mathrm{X}$ & $\mathrm{X}$ & $\mathrm{X}$ & $\mathrm{X}$ \\
\hline
\end{tabular}

A presença de MC-LR foi detectada em $100 \%$ das amostras analisadas (Tabela 2). Os valores oscilaram de $0,010 \mu \mathrm{g} . \mathrm{L}^{-1} \mathrm{em}$ fevereiro a $0,103 \pm 0,031 \mu \mathrm{g} . \mathrm{L}^{-1} \mathrm{em}$ abril, com média de $0,058 \pm 0,029$ $\mu \mathrm{g} . \mathrm{L}^{-1}$. Esta concentração máxima de MC-LR coincidiu com a maior densidade do gênero Microcystis (933 cel.mL ${ }^{-1}$ ) verificada no rio Tapajós neste mesmo período [48]. Isto implica que a elevada proliferação deste gênero potencialmente tóxico pode ter sido a principal causa do incremento de MC-LR na coluna d'água durante o mês de abril. Neste respectivo mês, inserido no início do período de águas altas conforme cota de inundação, foram registradas elevadas concentrações de nitrato que atingiu valor de $0,12 \pm 0,04 \mathrm{mg} . \mathrm{L}^{-1}$, enquanto o orto-fosfato mediu $0,02 \pm 0,01 \mathrm{mg} \cdot \mathrm{L}^{-1}$. Em abril quantificação da clorofila- $a$ foi de $2,06 \pm 0,16 \mu \mathrm{g} . \mathrm{L}^{-1}$, os sólidos totais dissolvidos foram $7,8 \mathrm{mg} . \mathrm{L}^{-1} \mathrm{e}$ a precipitação pluviométrica atingiu o seu maior valor médio de $471 \pm 117 \mathrm{~mm}$.

Tabela 2: Valores de microcistina-LR (MC-LR) no sistema no baixo rio Tapajós no período de outubro de 2010 a setembro de 2011.

\begin{tabular}{cc}
\hline Meses & Microcistina- LR $\left(\boldsymbol{\mu g} . \mathbf{L}^{-\mathbf{1}}\right)$ \\
\hline out/10 & 0,060 \\
$\mathrm{nov} / 10$ & 0,047 \\
$\mathrm{dez} / 10$ & 0,059 \\
$\mathrm{jan} / 11$ & 0,028 \\
$\mathrm{fev} / 11$ & 0,010 \\
$\mathrm{mar} / 11$ & 0,052 \\
$\mathrm{abr} / 11$ & 0,103 \\
$\mathrm{mai} / 11$ & 0,080 \\
jun/11 & 0,049 \\
jul/11 & 0,040 \\
ago/11 & 0,040 \\
set/11 & 0,086 \\
\hline Média $\pm \mathbf{D P}$ & $0,058 \pm 0,029$
\end{tabular}

O valor máximo de MC-LR encontrado no presente estudo representa $1 \%$ do valor máximo de $10 \mu \mathrm{g} . \mathrm{L}^{-1}$ indicado para exposição recreativa [22, 27]. No Canadá, nos EUA- Oklahoma e Ohioo valor de referência para águas recreacionais, é de $20 \mu \mathrm{g} . \mathrm{L}^{-1}$ de MC-LR [57]. A Califórnia, EUA, em comparação, utiliza $0,8 \mu \mathrm{g} \cdot \mathrm{L}^{-1}$ de MC-LR como limite para águas de uso recreativo. A Itália proíbe o banho a $25 \mu \mathrm{g} . \mathrm{L}^{-1}$ e a Alemanha recomenda o fechamento temporário de corpos d'água na presença de florações visíveis e se os níveis de MC-LR excederem $100 \mu \mathrm{g}$. $\mathrm{L}^{-1}$. Na Polônia, o valor de alerta básico para balneabilidade é de 2,5 $\mu \mathrm{g} . \mathrm{L}^{-1}$ [57].

Embora as concentrações de MC-LR do presente estudo tenham sido encontradas em água bruta, podem ocorrer ingestões da água acidentais pois o rio Tapajós é usado para fins recreativos. Diante desta possibilidade, os resultados de MC-LR do presente estudo foram confrontados com 
o valor máximo de microcistina $\left(1 \mu \mathrm{g} . \mathrm{L}^{-1}\right)$ estabelecido para potabilidade [29], e observou-se que a concentração máxima aqui verificada esteve abaixo do valor preconizado.

Os valores de MC-LR obtidos no presente estudo estiveram abaixo dos valores encontrados por Sá et al. (2010) [45] para amostras concentradas (> 0, $\left.3 \mu \mathrm{g} \cdot \mathrm{L}^{-1}\right)$ obtidas em análise pontual em duas estações amostrais no rio Tapajós mais próximas à área urbana da cidade de Santarém, em momento de densa floração de cianobactérias. Entretanto, estes mesmos autores, verificaram resultado negativo para a quantificação de MC-LR em água bruta do rio Tapajós, o que corrobora com os resultados obtidos no presente estudo. As concentrações de MC-LR determinadas neste estudo foram inferiores às encontradas na América do Sul, em ambientes de uso recreativo como na Argentina, rio Uruguai, com $0,6 \mu \mathrm{g} . \mathrm{L}^{-1}$ e rio Paraná, com $1,9 \mu \mathrm{g} . \mathrm{L}^{-1}$ [58]. No Uruguai as medidas de MC-LR em lagos às margens do rio Negro, foram no Palmar 2,6 mg. $\mathrm{L}^{-1}$, Bonete 2,0 mg. $\mathrm{L}^{-1}$ e Baygorria 20,04 mg. $\mathrm{L}^{-1}$ [35]. No Chile, o valor notificado no Lago Galindo foi 115,4 $\mu \mathrm{g} . \mathrm{L}^{-1}[59]$.

Neste estudo o regime pluviométrico foi caracterizado pelo agrupamento dos meses em dois períodos: 1) Período de estiagem (de outubro de 2010 a novembro de 2010, e de junho de 2011 a setembro de 2011) e 2) Período chuvoso (de dezembro de 2010 a maio de 2011). A concentração média de MC-LR no período de estiagem foi $0,054 \pm 0,019 \mu \mathrm{g} . \mathrm{L}^{-1}$ e de $0,062 \pm 0,039 \mu \mathrm{g} \cdot \mathrm{L}^{-1}$ no período chuvoso. Todavia não houve diferença estatística significativa quanto à sazonalidade (MC-LR- $\left.\mathrm{F}_{1,20}=0,48 ; \mathrm{p}=0,50\right)$ de acordo com a Análise de Variância Unifatorial $(\mathrm{p}<0,05 \%)$. Este comportamento difere de estudos realizados no estado do Pará, em ambientes lóticos na Ilha de Marajó, onde as detecções de MC-LR foram restritas a estação de estiagem [60].

A partir dos valores obtidos quanto ao nível do rio (cota), procedeu-se a caracterização do pulso de inundação da seguinte forma: 1) Período de Águas Baixas (de outubro de 2010 a novembro de 2010); 2) Período de Enchente (de dezembro de 2010 a março de 2011); 3) Período de Águas Altas (de abril a junho de 2011); e 4) Período de Vazante (de julho a setembro de 2011) (Figura $1)$.

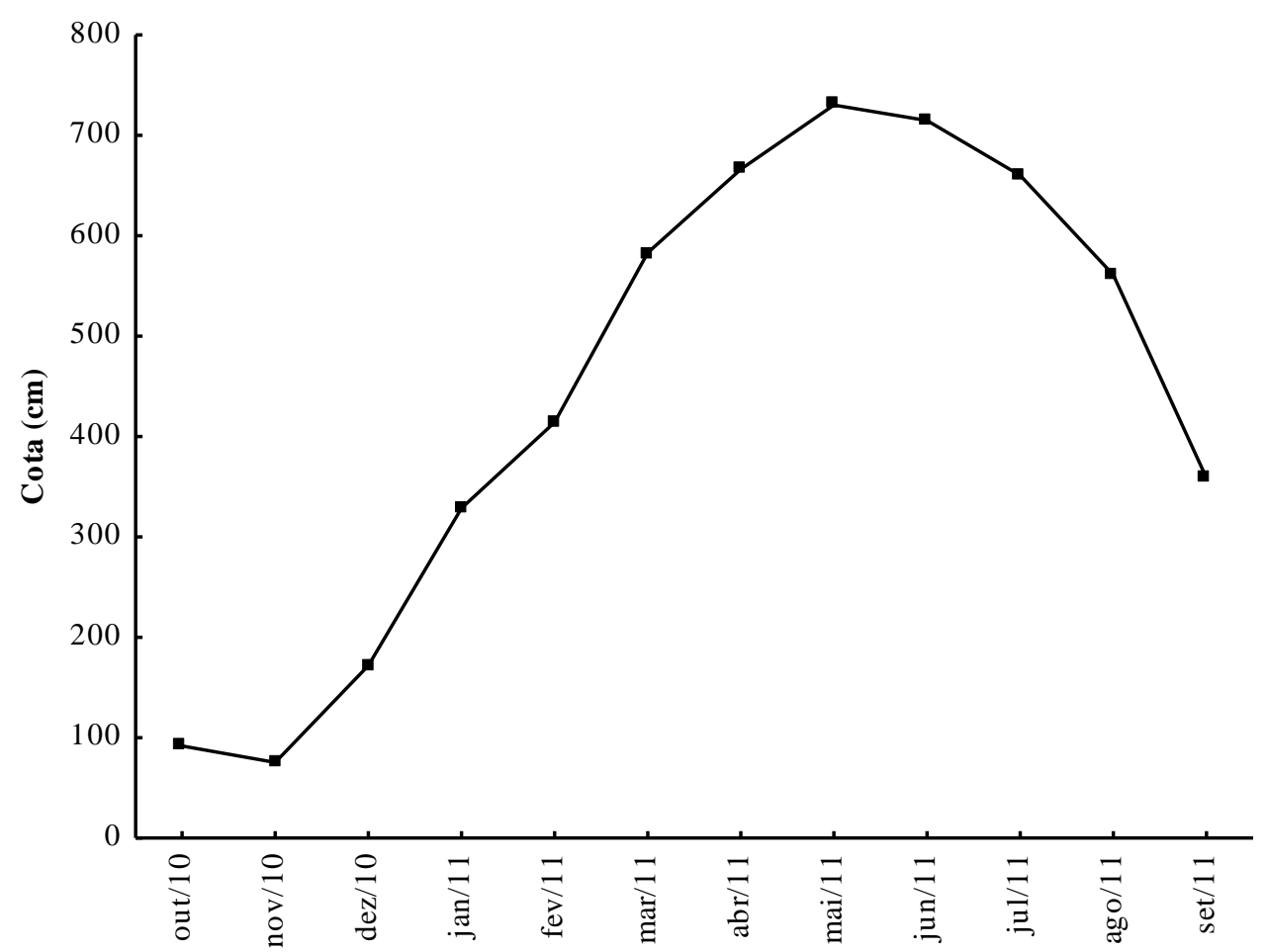

Figura 1: Nível médio (cota) mensal do rio Tapajós em sua área de drenagem no período de outubro de 2010 a setembro de 2011. Fonte: Agência Nacional de Águas.

Durante o período de águas baixas a concentração média de MC-LR foi $0,054 \pm 0,013 \mu \mathrm{g} . \mathrm{L}^{-1}$. Na enchente, o nível médio de MC-LR foi reduzido para $0,043 \pm 0,035 \mu \mathrm{g} . \mathrm{L}^{-1}$ voltando a subir 
durante o período de águas altas com média de $0,077 \pm 0,031 \mu \mathrm{g} . \mathrm{L}^{-1}$ e durante a vazante com concentração média de $0,055 \pm 0,024 \mu \mathrm{g} \cdot \mathrm{L}^{-1}$. Assim o padrão de distribuição das concentrações de MC-LR apresentou um leve acréscimo durante o período de águas altas e de vazante. No entanto, a Análise de Variância Unifatorial $(\mathrm{p}<0,05 \%)$ revelou não haver diferença estatística significativa nos níveis desta cianotoxina quanto ao pulso de inundação no rio Tapajós (Microcistina $-\mathrm{F}_{3,18}=1,50 ; \mathrm{p}=0,25$ ) (Figura 2). Dentre as variáveis biológicas investigadas no presente estudo, apenas a riqueza de cianobactérias foi influenciada significativamente pelo pulso de inundação. Isto se deve às alterações que este processo de oscilação periódica do nível do rio provoca nas variáveis físicas e químicas do rio Tapajós, a qual induz o desenvolvimento de espécies com diferentes formas de adaptação a estas oscilações periódicas [61].

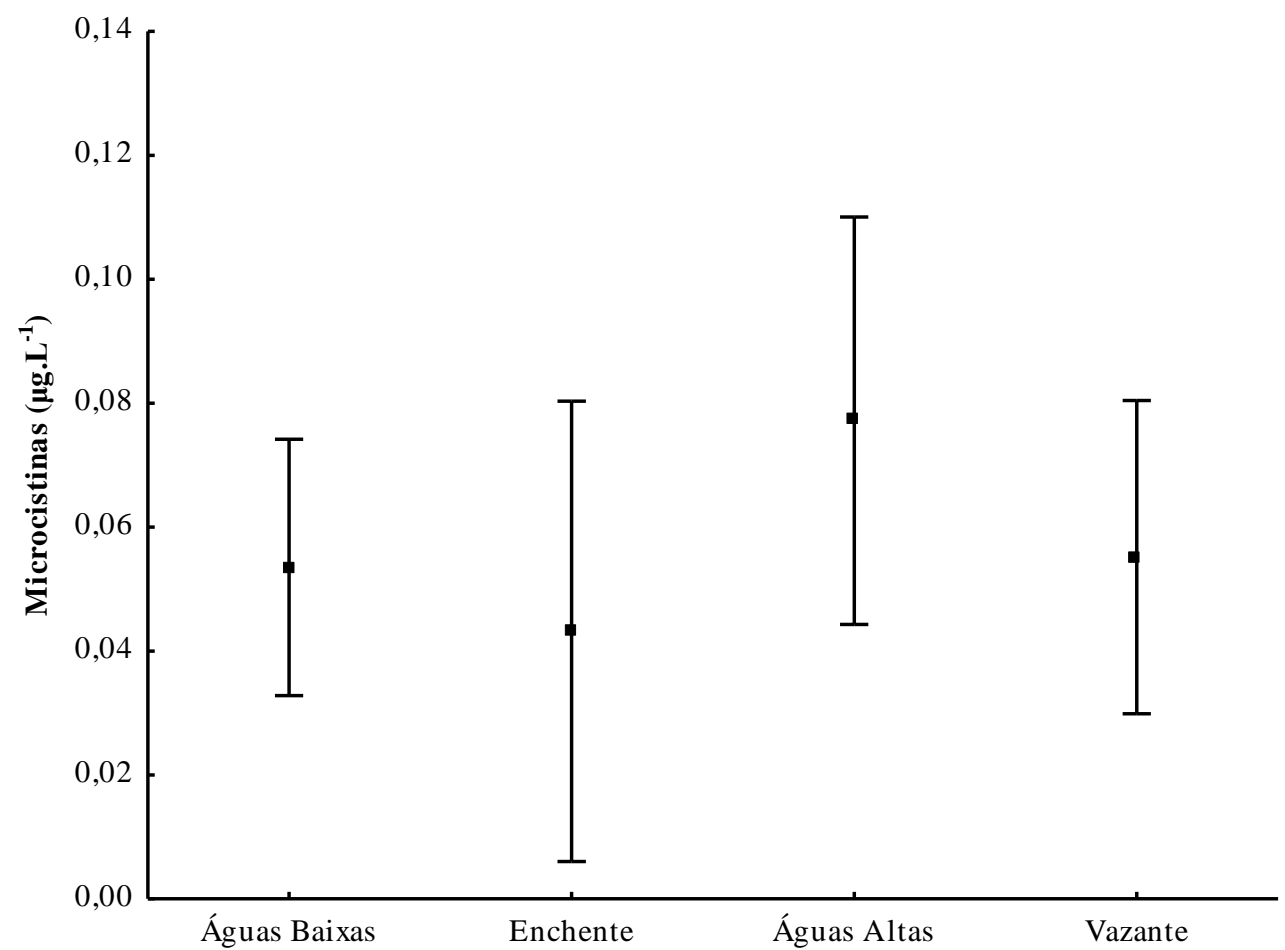

Figura 2:Flutuação das concentrações de microcistina-LR ao longo do pulso de inundação no rio Tapajós durante o período de outubro de 2010 a setembro de 2011.

O local estudado caracterizou-se por apresentar profundidade de extinção do disco de Secchi em torno de 2 metros, e baixos valores de condutividade elétrica e de nutrientes. A concentração de oxigênio dissolvido e os valores de temperatura da água apresentaram valores médios de 6,0 mg. $\mathrm{L}^{-1}$ e $29,5^{\circ} \mathrm{C}$, respectivamente. Enquanto os valores de $\mathrm{pH}$ estiveram em torno de 7. Já os valores de íons nitrogenados e orto-fosfato foram baixos, porém suportando valores de clorofilaa de até $6,9 \mathrm{mg} . \mathrm{L}^{-1}$. Na Tabela 3 pode ser observado os valores médios, por período do pulso de inundação, das variáveis limnológicas estudadas. Os valores mais elevados de nitrogênio (nitrito + nitrato) e orto-fosfato foram registrados no início do período águas altas, no mês de abril, quando foi registrado o maior valor médio de precipitação ao longo deste estudo. 
Tabela 3-Valores médios das variáveis analisadas correspondentes ao pulso de inundação no baixo rio Tapajós no período de outubro de 2010 a setembro de 2011.

\begin{tabular}{|c|c|c|c|c|c|c|c|c|c|c|c|}
\hline & $\begin{array}{l}\text { Transp. } \\
(\mathrm{m})\end{array}$ & $\begin{array}{l}\text { Cond. } \\
\left(\mu \mathrm{S} . \mathrm{cm}^{-1}\right)\end{array}$ & $\begin{array}{l}\text { STD } \\
\left(\mathrm{mg} . \mathrm{L}^{-1}\right)\end{array}$ & $\begin{array}{l}\text { OD } \\
\left(\mathrm{mg} \cdot \mathrm{L}^{-1}\right)\end{array}$ & $\begin{array}{l}\text { Temp. } \\
\left({ }^{\circ} \mathrm{C}\right)\end{array}$ & $\mathrm{pH}$ & $\begin{array}{l}\text { Amônia } \\
\left(\mathrm{mg}^{-L^{-1}}\right)\end{array}$ & $\begin{array}{l}\text { Nitrato } \\
\left(\mathrm{mg} \mathrm{L}^{-1}\right)\end{array}$ & $\begin{array}{l}\text { Nitrito } \\
\left(\mathrm{mg} . \mathrm{L}^{-1}\right)\end{array}$ & $\begin{array}{l}\text { Fosf. } \\
\left(\mathrm{mg} . \mathrm{L}^{-1}\right)\end{array}$ & $\begin{array}{l}\text { Clorof.a } \\
\left(\text { ug.L } L^{-1}\right)\end{array}$ \\
\hline $\begin{array}{l}\text { Águas } \\
\text { Baixas }\end{array}$ & 1,4 & 33,6 & 16,8 & 5,5 & 30,5 & 7,4 & 0,00 & 0,02 & 0,01 & 0,01 & 3,42 \\
\hline Enchente & 1,6 & 50,6 & 26,0 & 5,8 & 28,0 & 7,2 & 0,13 & 0,15 & 0,01 & 0,02 & 6,91 \\
\hline $\begin{array}{l}\text { Águas } \\
\text { Altas }\end{array}$ & 1,8 & 15,9 & 8,2 & 6,1 & 29,4 & 6,9 & 0,01 & 0,07 & 0,01 & 0,01 & 1,47 \\
\hline Vazante & 2,6 & 16,1 & 8,1 & 6,5 & 30,3 & 7,3 & 0,00 & 0,01 & 0,00 & 0,00 & 5,58 \\
\hline $\begin{array}{l}\text { Média } \\
\pm \mathrm{DP}\end{array}$ & $1,9 \pm 0,6$ & $28,7 \pm 31,7$ & $14,6 \pm 16,2$ & $6,0 \pm 0,9$ & $29,5 \pm 2,2$ & $7,2 \pm 0,5$ & $0,04 \pm 0,08$ & $0,06 \pm 0,09$ & $0,01 \pm 0,01$ & $0,01 \pm 0,01$ & $4,43 \pm 4,78$ \\
\hline
\end{tabular}

Legenda: Transp.: Transparência; Cond.: Condutividade elétrica; STD: Sólidos Totais Dissolvidos; OD: Oxigênio Dissolvido; Temp.: Temperatura; pH: potencial Hidrogeniônico; Fósf.: Orto-fosfato; Clorof. a: Clorofila- $a$. 
A Correlação Linear de Pearson ( $\mathrm{p}<0,05 \%)$ foi efetuada a partir dos valores médios mensais das variáveis físicas e químicas com a finalidade de avaliar o efeito isolado de cada uma das variáveis investigadas sobre os níveis de MC-LR. Os resultados evidenciaram que nenhuma destas se correlacionou isoladamente com os níveis de MC-LR, exceto a clorofila- $a$. Houve correlação negativa entre os níveis de clorofila- $a$ e os níveis de MC-LR ( $r=-0,53)$. Portanto, enquanto a biomassa de cianobactérias, representada pela clorofila- $a$ foi mantida, as concentrações de MC-LR foram baixas (Figura 2 e Tabela 3). Todavia, após a morte e lise celular de cianobactérias ocorreu a liberação das cianotoxinas para o ambiente. Este aumento de MC-LR concomitante com o declínio dos níveis de clorofila- $a$, é um acontecimento comum segundo Calijuri et al. (2006) [11]. Dessa maneira concentrações menores de clorofila- $a$ indicando menor presença do fitoplanctônica não correspondem necessariamente a ausência de MC-LR no ambiente aquático.

Análise de Regressão Linear Múltipla $(\mathrm{p}<0,05 \%)$ gerou o modelo explicativo da influência conjunta das variáveis independentes sobre os níveis de MC-LR. Em ordem decrescente de importância, o modelo demonstrou o efeito simultâneo da interação entre as seguintes variáveis: amônia, nitrato, oxigênio dissolvido, nitrito e orto-fosfato $(\mathrm{R} 2=0,70 ; \mathrm{p}=0,001)$ :

$\mathrm{Y}=(\mathrm{MC}-\mathrm{LR})=0,098+(-1,4 *$ amônia $)+(1,07 *$ nitrato $)+(0,53 *$ oxigênio dissolvido $)+$ $(0,31 *$ nitrito $)+(0,24 *$ orto-fosfato $)+$ resíduo

Conforme este modelo, a sinergia do aporte de nitrato, nitrito, fosfato e a diminuição de amônia na coluna d'água somados a presença de oxigênio regulou $70 \%$ da flutuação dos níveis de MCLR no rio Tapajós.

A amônia é a principal forma inorgânica de nitrogênio prontamente utilizável pelo fitoplâncton, além disso, ao ser introduzido no sistema este íon é rapidamente transformado em nitrito e nitrato pelo processo de nitrificação o qual requer condição de aerobiose [62]. Segundo estudos, células tóxicas de Microcystis, gênero presente em todo o período amostral, parecem ter uma necessidade maior de $\mathrm{N}$ e $\mathrm{P}$ do que as células não tóxicas [63]. Além disso, há evidências de que o aumento da carga de nitrogênio pode aumentar a quantidade de cianobactérias não fixadoras de nitrogênio, tais como Microcystis e Planktothrix [7].

Algumas espécies de cianobactérias são capazes de fixar nitrogênio atmosférico (diazotróficas), sendo favorecidas durante a limitação de nitrogênio no ambiente aquático em detrimento de outros grupos fitoplanctônicos [64, 65]. O gênero Dolichospermum, encontrado em $75 \%$ dos meses amostrados, é reconhecido como produtor de heterocistos, estruturas celulares capazes de fixar nitrogênio atmosférico $[66,67,68]$. O gênero Synechococus também fixador de nitrogênio atmosférico, porém sem heterocisto [69], esteve presente em todo o período amostrado. Desta maneira, estes gêneros em virtude de sua capacidade de obtenção de nitrogênio em períodos de maior escassez, se sobressaem em relação ao fitoplâncton geral. Entretanto, dados taxonômicos não podem ser usados de maneira confiável para determinar a toxicidade em cianobactérias, porque dentro de uma espécie existem toxigênicos e cepas não-toxigênicas, dependendo da presença e expressão de genes reguladores da produção de toxinas [2].

Nos últimos anos, ações antrópicas como a mineração, o avanço da fronteira agrícola, o desmatamento e o crescimento demográfico têm se acentuado ao longo da bacia de drenagem do rio Tapajós [37, 39]. Por conseguinte, a retirada da vegetação natural da bacia e a substituição por áreas agrícolas fertilizadas, colaboram para a redução na capacidade de infiltração do solo fazendo com que os nutrientes, já adicionados em excesso na agricultura, tendam a escoar até atingir o corpo d'água [70]. A partir das observações relatadas neste estudo, infere-se que após eventos de chuva, mais frequentes no período de enchente/início do período de águas altas, ocorre o carreamento para o rio de matéria orgânica proveniente de fontes difusas da área de drenagem da bacia do rio Tapajós. Assim, o enriquecimento nutricional, advindo destes processos humanos colaboram para o desenvolvimento de florações de cianobactérias potencialmente tóxicas no baixo rio Tapajós. Isto pode ser observado pela dominância de cianobactérias a qual tem sido notificada no trecho inferior do rio Tapajós nos últimos anos [38, 45, 48]. É importante notar que há consenso de que as proliferações de cianobactérias tóxicas são eventos complexos, e não são causados por um único fator ambiental, mas sim por múltiplos fatores ocorrendo simultaneamente [12], inclusive podendo está associado ao aumento da disponibilidade bicabornato na água 
advindo do dióxido de carbono atmosférico que pode favorecer o crescimento de cianobactérias potencialmente tóxica $[40,41]$.

\section{CONCLUSÃO}

Embora em concentrações abaixo do valor recomendado para o contato em uso recreacional, a presença de MC-LR foi detectada em todo o período amostral. As maiores concentrações de MCLR coincidiram com a maior densidade populacional das espécies de Microcystis notificado no rio Tapajós por Silva (2012) [48]. Do total de 11 táxons de cianobactérias registrados neste estudo, três gêneros apresentam histórico na produção de MC-LR: Microcystis, Dolichospermum e Planktothrix. E o gênero Microcystis, com as espécies M. aeruginosa e M. wesenbergii, esteve presente em todo o período amostral. Temporalmente, não houve diferença estatística significativa nos níveis de MC-LR e o regime pluviométrico e pulso de inundação no rio Tapajós.

A ocorrência de gêneros de cianobactérias potencialmente tóxicas, somados a detecção de MCLR em todo o período de estudo é um alerta para atenção à saúde pública dadas as possibilidades de exposição continuada do potencial hepatotóxico. Além disso, o possível contato com dermatotoxinas, via uso recreacional no rio Tapajós é relevante. Estas substâncias são componentes estruturais da parede celular de todas as cianobactérias e conferem riscos à saúde causados pela exposição por inalação de aerossóis, ingestão acidental e pelo contato dérmico. Desta forma, crianças, idosos e imunodeprimidos são os mais suscetíveis a exposição em atividades recreativas durante momentos com formação de "escuma", no rio Tapajós.

\section{AGRADECIMENTOS}

Ao Laboratório de Biologia Ambiental da Universidade Federal do Oeste do Pará-UFOPA pelo suporte nas análises físico-químicas, ao curso de mestrado em Recursos Naturais da Amazônia e ao curso doutorado em Sociedade, Natureza e Desenvolvimento pelo apoio logístico e, a Fundação Amazônia de Amparo a Estudos e Pesquisas (FAPESPA) pela bolsa de estudo.

\section{REFERÊNCIAS BIBLIOGRÁFICAS}

1. Brasil. Ministério da Saúde. Cianobactérias tóxicas na água para consumo humano na saúde pública e processos de remoção em água para consumo humano. Fundação Nacional de Saúde-FUNASA, Brasília, DF; 2003. 56 p.

2. Pick FR. Blooming algae: a Canadian perspective on the rise of toxic cyanobacteria. Can J Fish Aquat. Sci. 2016 Apr; 73(7): 149-1158, doi:10.1139/cjfas-2015-0470.

3. Ferrão-Filho AS. Bioacumulação de cianotoxinas e seus efeitos em organismos aquáticos. Oecol Brasil. 2009;13(2):272-312.

4. Calado SLM, Santos GS, Wojciechowski J, Magalhães VF, Assis HCS. The accumulation dynamics, elimination and risk assessment of paralytic shellfish toxins in fish from a water supply reservoir. Sc Total Environ. 2019 Feb;651(1):3222-3229, doi:10.1016/j.scitotenv.2018.10.046.

5. Paerl HW, Huisman J. Climate change: a catalyst for global expansion of harmful cyanobacterial blooms. Environ Microbiol. 2009 Feb; 1(1): 27-37, doi:10.1111/j.1758-2229.2008.00004.

6. Chorus I, Bartram J. Toxic cyanobacteria in water: a guide to their public health consequences, monitoring and management. Londres: E. \& F.N. SPON; 1999. 400 p.

7. Huisman J, Codd GA, Paerl HW, Ibelings BW, Verspagen JMH, Visser PM. Cyanobacterial blooms. Nat Rev Microbiol. 2018 Aug;16(8):471-483, doi: 10.1038/s41579-018-0040-1.

8. Carmichael WW. Health effects of Toxin-Producing Cyanobacteria: "The CyanoHABs". Human and Ecological Risk Assessment. 2001 Set;7(5):1393-1407, doi: 10.1080/20018091095087.

9. Leflaive J, Ten-Hage L. Algal and cyanobacterial secondary metabolites in freshwaters: a comparison of allelopathic compounds and toxins: special review. Freshwater Biol. 2007 Fev; 52(1):199-214, doi: 10.1111/j.1365-2427.2006.01689.

10. Oliveira VR, Carvalho GMC, Casquilho NV, Moreira-Gomes MD, Soares RM, Azevedo SMFO, Lima LM, Barreiro EJ, Takiya CM, Zin WA. Lung and liver responses to 1- and 7-day treatments with LASSBio-596 in mice subchronically intoxicated by microcystin-LR. Toxicon. 2018 Jan;141(1):1-8, doi:10.1016/j.toxicon.2017.10.029.

11. Calijuri MC, Alves MSAA, Santos ACA. Cianobactérias e cianotoxinas em águas continentais. São Carlos: RiMa; 2006. 118 p. 
12. O’Neil JM, Davis TW, Burford MA, Gobler CJ. The rise of harmful cyanobacteria blooms: The potential roles of eutrophication and climate change. Harmful Algae. 2012 Feb; 14(1):313334,.doi:10.1016/j.hal.2011.10.027.

13. Ruvieri V, Shundo L, Alaburda J, Sabino M. Microcistinas em água de hemodiálise de clínicas do estado de São Paulo. Rev Inst Adolfo Lutz. 2004 Jan;63(2):220-223.

14. Pham T, Utsumi M. An overview of the accumulation of microcystins in aquatic ecossystems. J Environ Manag. 2018 May; 213(1):520-529, doi:10.1016/j.jenvman.2018.01.077.

15. Soares RM. Toxicologia de Cianotoxinas: Microcistinas as Estrelas do Tema. Oecol Brasil. 2009 Jun;13(2):259-271, doi: 10.4257/oeco.2009.1302.04.

16. Puddick J, Prinsep MR, Wood SA, Kaufononga SAF, Cary SC, Hamilton DP. High levels of structural diveristy observed in microcystins from Microcystis CAWBG11 and characterization of six new microcystin congeners. Mar Drugs. 2014 Nov;12(11):5372-5395, doi: 10.3390/md12115372.

17. Puddick J, Prinsep MR, Wood SA, Cary SC, Hamilton DP, Holland PT. Further characterization of glycine-containing microcystins from the McMurdo dry Valleys of Antarctica. Toxins. 2015 Feb;7(2):493-515, doi: 10.3390/toxins7020493.

18. Sivonen K. Toxins produced by cyanobacteria. In: Miraglia M, Van Egmond HP, Brera C, Gilbert J. Mycotoxins and Phycotoxins - Development in Chemistry, Toxicology and Food Safety. EUA; 1998. p. 547-586.

19. Buratti FM, Manganelli M, Vichi S, Stefanelli M, Scardala S, Testai E, Funari E. Cyanotoxins: producing organisms, occurrence, toxicity, mechanism of action and human health toxicological risk evaluation. Arch Toxicol. 2017 Mar;91(1):1049-1130, doi: 10.1007/s00204-016-1913-6.

20. Lowe J, Souza-Menezes J, Freire DS, Mattos LJ, Castiglione RC, Barbosa CML, Santiago L, Ferrão FM, Cardoso LHD, Silva RT, Vieira-Beiral HJ, Vieyra A, Morales MM, Azevedo SMFO, Soares RM. Single sublethal dose of microcystins-LR is responsible for different alterations in biochemical, histological and physiological renal parameters. Toxicon. 2012 May;59(6):601-609, doi: 10.1016/j.toxicon.2012.02.003.

21. Carvalho GM, Oliveira VR, Casquilho NV, Araujo AC, Soares RM, Azevedo SM, Pires KM, Valença SS, Zin WA. Pulmonary and hepatic injury after sub-chronic exposure to sublethal doses of microcystinLR. Toxicon. 2016 Mar;112(1):51-58, doi:10.1016/j.toxicon.2016.01.066.

22. Chorus I, Falconer IR, Salas HJ, Bartram J. Health risks caused by freshwater cyanobacteria in recreational waters. J Toxicol Environ. Health-Part B - Crit Rev 2000 Nov;3(4):323-347, doi:10.1080/109374000436364.

23. Backer LC, McNeel SV, Barber T, Kirkpatrick B, Williams C, Irvin M, Zhou Y, Johnson TB, Nierenberg K, Aubel M, LePrell R, Chapman A, Foss A, Corum S, Hill VR, Kieszak SM, Cheng YS. Recreational exposure to microcystins during algal blooms in two california lakes. Toxicon. 2010 May;55(5):909-921, doi:10.1016/j.toxicon.2009.07.006.

24. Funari E, Manganelli M, Buratti FM, Testai E. Cyanobacteria blooms in water: Italian guidelines to assess and manage the risk associated to bathing and recreational activities. Sci Total Environ. 2017 Nov;598(1):867-880, doi:doi.org/10.1016/j.scitotenv.2017.03.232.

25. Neumann AC, Melnik S2, Niessner R, Stoeger E, Knopp D. Microcystin-LR Enrichment from Freshwater by a Recombinant Plant-derived Antibody Using Sol-Gel-Glass Immunoextraction. Anal Sci. 2019 Feb;35(2):207-214, doi: 10.2116/analsci.18P384.

26. Oliveira VR, Mancin VGL, Pinto EF, Soares RM, Azevedo SMFO, Macchione M, Carvalho AR, Zin WA. Repeated intranasal exposure to microcystin-LR affects lungs but not nasal epithelium in mice. Toxicon. 2015 Sep;104(1):14-18, doi:10.1016/j.toxicon.2015.07.331.

27. Farrer D, Contador M, Hillwige R, Cude C. Health-Based Cyanotoxin Guideline Values Allow for Cyanotoxin-Based Monitoring and Efficient Public Health Response to Cyanobacterial Blooms. Toxins. 2015 Fev;7(2):457-477, doi:10.3390/toxins7020457.

28. WHO - World Health Organization. Guidelines for drinking-water quality: fourth edition incorporating the first addendum. Geneva: World Health Organization; 2017, 631p. Disponível em: <https://apps.who.int/iris/bitstream/handle/10665/254637/9789241549950-

eng.pdf;jsessionid=DAF7E9CDAEB5C2B5031FC70718D9AB55?sequence=1>. Acesso em 04/mar./19.

29. BRASIL. Ministério da Saúde. Portaria de Consolidação no 5, de 28 de setembro de 2017, Anexo XX. Dispõe sobre a Consolidação das normas sobre as ações e os serviços de saúde do Sistema Único de Saúde. Diário oficial da União. 2017, p.926.

30. BRASIL. Ministério do Meio Ambiente. Conselho Nacional do Meio Ambiente- CONAMA. Resolução $n^{\circ} 274,29$ de novembro 2000. Define os critérios de balneabilidade em águas brasileiras. Diário Oficial da União-DOU, 2001, p. 3. 
31. BRASIL. Ministério do Meio Ambiente. Conselho Nacional do Meio Ambiente- CONAMA. Resolução $n^{\circ} 357$, de 17 de março de 2005. Dispõe sobre a classificação dos corpos de água e diretrizes ambientais para o seu enquadramento, bem como estabelece as condições e padrões de lançamento de efluentes, e dá outras providências. Diário Oficial da União-DOU, 2005, p. 23.

32. Sant'Anna CL, Azevedo MTP, Agujaro LF, Carvalho MC, Carvalho LR, Souza RCR. Manual ilustrado para identificação e contagem de cianobactérias planctônicas de águas continentais brasileiras. Rio de Janeiro: Interciência; 2006. 58 p.

33. Soares RM, Cagido VR, Ferraro RB, Meyer-Fernandes JR, Rocco PRM, Zin WA, Azevedo SMFO. Effects of microcystin-LR on mouse lungs. Toxicon. 2007 Set;50(3):330-338, doi: 10.1016/j.toxicon.2007.04.003.

34. Bittencourt-Oliveira MC, Piccin-Santos V, Moura NA, Aragao-Tavares NKC, Cordeiro-Araujo M K. Cyanobacteria, microcystins and cylindrospermopsin in public drinking supply reservoirs of Brazil. Anais Academia Bras Ci. 2014 Mai;86(1):297-309, doi: 10.1590/0001-3765201302512.

35. Bonilla S, Haakonsson S, Somma A, Gravier A, Britos A, Vidal L, De León L, Brena BM, Pírez M, Piccini C, Martínez De La Escalera G, Chalar G, Gonzalez-Piana M, Martigani F, Aubriot L. Cianobacterias y cianotoxinas en ecossistemas límnicos de Uruguay. INNOTEC. 2015 Out;10(1):9-22.

36. Dorr FA, Pinto E, Soares RM, Azevedo SMFO. Microcystins in South American aquatic ecosystems: occurrence, toxicity and toxicological assays. Toxicon. 2010 Dez;56(7):1247-1256, doi: 10.1016/j.toxicon. 2010.03.018.

37. Brasil. Ministério da Economia. Instituto Brasileiro de Geografia e Estatística-IBGE. Disponível em <https://www.ibge.gov.br/geociencias-novoportal/cartas-e-mapas/mapas-regionais/15976-fronteiraagricola-amazonia-legal.html?=\&t=o-que-e>. Acesso em: 15/fev./19.

38. Lobo FL, Costa M, Novo EMLM, Telmer K. Effects of Small-Scale Gold Mining Tailings on the Underwater Light Field in the Tapajós River Basin, Brazilian Amazon. Remote Sens. 2017 Aug;9(8):861. doi:10.3390/rs9080861.

39. Instituto do Homem e Meio Ambiente da Amazônia -Imazon. Desmatamento e Degradação Florestal de Santarém. $\quad$ Disponível em <https://imazon.org.br/PDFimazon/Portugues/outros/10_Folder_Santarem_web.pdf > Acesso em $18 /$ fev./19.

40. Sandrini G, Ji X, Verspagen JMH, Tann RP, Slot PC, Luimstra VM, Schuurmans JM, Matthijs HCP, Huisman J. Rapid adaptation of harmful cyanobacteria to rising CO2. Proceedings of the National Academyof Sciences of the United States of America. 2016 Aug; 113(33):9315-9320 doi: 10.1073/pnas.1602435113.

41. Brito MTS, Duarte-Neto PJ, Molica RJR. Cylindrospermopsis raciborskii and Microcystis aeruginosa competing under different conditions of $\mathrm{pH}$ and inorganic carbon. Hydrobiologia. 2018 Jun;815(1):253266. doi:10.1007/s10750-018-3567-2

42. Sant'Anna CL, Azevedo MTP. Contribution to the knowledge of potentially toxic cyanobacteria from Brazil. Nova Hedwigia. 2000 Nov;71(3):359-385.

43. Vieira JMS, Azevedo MTP, Azevedo SMFO, Honda RY, Corrêa B. Toxic cyanobacteria and microcystin concentrations in a public water supply reservoir in the Brazilian Amazonia region. Toxicon. 2005 Jun;45(7):901-909, doi:10.1016/j.toxicon.2005.02.008.

44. Vieira JMS, Azevedo MTP, Azevedo SMFO, Honda RY, Corrêa B. Microcystin production by Radiocystis fernandoi (Chroococcales, Cyanobacteria) isolated from a drinking water reservoir in the city of Belém, PA, Brazilian Amazonia region. Toxicon. 2003 Dez;42(7):709-713, doi:10.1016/j.toxicon.2003.08.004.

45. Sá LLC, Vieira JMS, Mendes RA, Pinheiro SCC, Vale ER, Alves FAZ, Jesus IM, Santos ECO, Costa VB. Ocorrência de uma floração de cianobactérias tóxicas na margem direita do rio Tapajós, no Município de Santarém (Pará, Brasil). Pan-Amaz. Saúde. 2010 Mar;1(1):159-166, doi 10.5123/S217662232010000100022.

46. Sioli H. Amazônia: fundamentos da ecologia da maior região de florestas tropicais. Petrópolis: Vozes; 1995. 72 p.

47. Cunha HB, Pascoaloto D. Hidroquímica dos Rios da Amazônia. Manaus: Centro Cultural dos Povos da Amazônia; 2006. 147 p.

48. Silva SCF. Ecotoxicologia de cianobactérias no canal principal do Baixo Rio Tapajós, Santarém, Pará, Amazônia, Brasil. Santarém (PA): Universidade Federal do Oeste do Pará; 2012.93 p.

49. Rossetti DF. The role of tectonics in the late Quaternary evolution of Brazil's Amazonian landascape. Earth-Science Rev. 2014 Dez;139(1):362-389, doi:10.1016/j.earscirev.2014.08.009.

50. Uherkovich, G. Algen aus den Flüssen Rio Negro und Rio Tapajós. Amazoniana. 1976 Abr;5(4):465515. 
51. Schmidt GW. Primary Production of Phytoplankton in the Three Types of Amazonian Water. Amazoniana. 1982 Oct;7(3):335-348.

52. AWWA - American Water Works Association. Standard methods for examination of water and wasteWater. 18Th. Washington: American Public Health Association; 2005. 926 p.

53. Strickland JDH, Parsons TR. A practical handbook of seawater analysis. Canada: Fisheries Research Board; 1968.311 p.

54. Soares RM, Azevedo SMFO, Fan TS, Skoczenki B. Análise de eficiência de Kits de tubo de ELISA comerciais para a detecção de microcistina em água bruta e tratada de várias regiões brasileiras. Beacon Analytical Systems Inc. Disponível em: https://slideplayer.com.br/slide/3150032/ Acesso em $07 /$ jun/2019.

55. Chu FS, Huang X, Wei RD, Carmichael WW. Production and Characterization of Antibodies Against Microcystins. Applied Environ Microbiol. 1989 Ago;55(8):1928-1933.

56. Sant'anna CL, Azevedo MTP, Werner VR, Dogo CR, Rios FR, Carvalho LR. Review of toxic species of Cyanobacteria in Brazil. Algological Studies. 2008 Abr;126(1):251-265, doi: 10.1127/18641318/2008/0126-0251.

57. Aguilera A, Haakonsson S, Martin MV, Salerno GL, Echenique RO. Bloom-forming cyanobacteria and cyanotoxins in Argentina: A growing health and environmental concern. Limnologica. 2018 Dez;69(1):103-114, doi:10.1016/j.limno.2017.10.006.

58. Ibelings BW, Backer L, Kardinaal WE, Chorus I. Current approaches to cyanotoxin risk assessment and risk management around the globe. Harmful Algae. 2014 Dez;40(1):63-74, doi: 10.1016/j.hal.2014.10.002.

59. Almanza V, Parra O, Bicudo CEM, Baeza C, Beltran J, Figueroa R, Urrutia R. Occurrence of toxic blooms of Microcystis aeruginosa in a central Chilean $\left(36^{\circ}\right.$ Lat. S) urban lake. Rev Chilena Historia Nat. 2016 Apr; 89(1):8-12, doi:10.1186/s40693-016-0057-7.

60. Leal AC, Soares MCP, Silva CAM, Alves MM, Cartágenes PRB, Bensabath G. Níveis da hepatotoxina Microcistina em ambientes aquáticos de área rural, Ilha do Marajó, Pará, Brasil. Rev Sociedade Bras Medic Trop. 1999.32 (supl):448.

61. Junk WJ, Bayley PB, Sparks RE. The flood pulse concept in river floodplain systems. Can Spec Publ Fish Aquat Sci. 1989;106(1):110-127.

62. Esteves FA. Fundamentos de Limnologia. Rio de Janeiro: Interciência; 2011. 826 p.

63. Davis TW, Harke MJ, Marcoval MA, Goleski J, Orano-Dawson C, Berry DL, Gobler CJ. Effects of nitrogenous compounds and phosphorus on the growth of toxic and non-toxic strains of Microcystis during cyanobacterial blooms. Aquat Microb Ecol. 2010 Out;61(1):149-162, doi:10.3354/ame01445.

64. Komárek J. Coccoid and Colonial Cyanobacteria. In: Wehr, J.D. e Sheath, R.G. (Ed.) Freshwater Algae of North America. EUA: Academic Press; 2003. p. 59-116.

65. Silverman SN, Kopf SH, Bebout BM, Gordon R, Som SM. Morphological and isotopic changes of heterocystous cyanobacteria in response to $\mathrm{N}_{2}$ partial pressure. Gebiology. 2019Jan;17(1):60-75, doi:10.1111/gbi.12312.

66. Kumar K, Mella-Herrera RA, Golden JW. Cyanobacterial heterocysts. Cold Spring Harb Perspect Biol. 2010;2(4):a000315, doi:10.1101/cshperspect.a000315.

67. Wang S, Xiao J, Wan L, Zhou Z, Wang Z, Song C, Zhou Y, Cao X. Mutual Dependence of Nitrogen and Phosphorus as Key Nutrient Elements: One Facilitates Dolichospermum flos-aquae to Overcome the Limitations of the Other. Environ. Sci. Technol. 2018 May;52(10):5653-5661, doi:10.1021/acs.est.7b04992.

68. Kapkov VI, Vasilieva SG, Lobakova ES. Growth of Toxic Cyanobacteria Dolichospermum flos-aquae (Anabaena flos-aquae) in the Waters of the Boreal Zone. Moscow Univ Biol Sci Bull. 2019 Jan; 74(1)15-20, doi:10.3103/S0096392519010036.

69. Tsujimoto R, Kamiya N, Fujita Y. Transcriptional regulators ChlR and CnfR are essential for diazotrophic growth in nonheterocystous cyanobacteria. Proc Natl Acad Sci U S A. 2014 May;111(18):6762-6767, doi: 10.1073/pnas.1323570111.

70. Von Sperling M. Introdução à qualidade das águas e tratamento dos esgotos. Belo Horizonte: Departamento de Engenharia Sanitária e Ambiental- Universidade Federal de Minas Gerais; 2005. 452 p. 